\section{9 HOW TO PREVENT CHOKING INJURIES IN PAEDIATRIC AGES}

'Dario Gregori, '2Luca Rosati, ${ }^{2}$ Solidea Baldas, 'Giulia Lorenzoni, ${ }^{3}$ Hugo Rodriguez. ${ }^{1}$ University of Padua, Italy; ${ }^{2}$ Prochild NPO Trieste, Italy; ${ }^{3}$ Paediatric Hospital Garrahan, Buenos Aires, Argentina

\subsection{6/injuryprev-2016-042156.179}

Background Choking injuries are one of the leading causes of death in paediatric ages. In recent years, several initiatives have been taken to reduce the burden of this phenomenon.

The major burden of food injuries, representing between $70 \%-85 \%$ of the overall cases, has no countermeasures.

Objective A MOOC (Massive Open online course) for disseminating information on these injuries, has been realised. The project consists of a series of informative videos freely available on a dedicated website with a distinctly international feel, available in the most spoken languages. The video contents are realised on the basis of data provided by the Susy Safe registry, which is an International registry of foreign body injuries in children aged 014 for collecting and analysing injury data and highlighting risks associated with consumer products. Currently, the registry has collected over 25.000 cases.

Presently Food bones, Nuts and Seeds and grains have the highest incidence, nevertheless, less frequent but more severe injuries, which can cause death, are those due to meat, sweets and other foods which share certain characteristics: shape, size, and consistency.

Results The videos provide basic information addressed to those who have the task of preparing food for children, explaining in a clear and as simple as possible manner how to reduce the risk presented by certain foods.

In each video specific topics on food choking in children are clearly addressed covering also different aspects of the problem and they are presented by a field expert.

Main objective of the project is to prevent injuries caused by food but also to disseminate information on this issue.

Conclusions The data show a lack of information and the need to implement specific educational campaigns targeted to families and supervisors, free and accessible to anyone, capable of reaching people from diverse ethnic, social and cultural backgrounds including the disadvantaged.

\section{FALLS IN CHILDREN UNDER ONE YEAR}

${ }^{1,2}$ Christopher S Mulligan, ${ }^{2} J u l i e$ Brown, 1,2 2 usan Adams. 'Sydney Children's Hospital, Australia; ${ }^{2}$ Neuroscience Research Australia, Randwick, Australia

\subsection{6/injuryprev-2016-042156.180}

Background Falls in children are a common cause of injury and hospital presentation. Compared to older children, infants under age one are likely to have distinctive causation and injury patterns of falls as they are generally not yet fully independently mobile and falls are more directly the responsibility of the caregiver. There is little published on the relevant predictors of injury, mechanistic factors and injury patterns in this age group.

Methods We conducted a retrospective study of children 12 months and under who presented after a fall to a paediatric trauma centre in Sydney, Australia. The circumstances and mechanisms of the fall, injury patterns, burden of investigations and outcomes were analysed.

Results Over a 3 year period (2011-13), a total of 916 children under 1 presented following a fall. 110 were admitted and there was one death. All admitted cases involved a short distance fall. The most common mechanisms were children falling from furniture, baby equipment and being dropped by adults. Patients dropped by others were 3 times more likely to be admitted than children presenting following other fall types. Patients who fell from furniture had significantly longer hospital stays.

Head injury was the most common reason for admission $(85 \%)$. Twenty patients had severe injuries (Abbreviated Injury Score $>15$ ). There were 7 neurosurgical and 12 orthopaedic/general surgical operations performed. There was no association between injury mechanism and severity. Twenty patients were referred to the Child Protection Unit for investigation and the rate of non-accidental injury in admitted patients was 5\%.

Conclusions Fall mechanisms involving children being dropped by adults, and falls from cots, beds or couches carry the highest clinical burden. These mechanisms should be targets for injury prevention and inform the design of safe equipment and environments for babies.

\section{DEVELOPING EDUCATIONAL PACKAGE ON CHILD INJURY PREVENTION MOBILISING WOMENS' GROUPS IN RURAL NEPAL}

${ }^{1}$ Puspa Raj Pant, ${ }^{2}$ Matthew Ellis, ${ }^{1}$ Toity Deave, ${ }^{1}$ Julie Mytton. ${ }^{1}$ University of the West of England, Bristol; ${ }^{2}$ University of Bristol

\subsection{6/injuryprev-2016-042156.181}

Background Non-fatal injuries are many times more common than fatal injuries and may lead to lifelong consequences in children. Lack of child injury surveillance system hinders evidence generation in a low-income setting. Children in rural Nepal are exposed to widespread injury risks and families may have little awareness of how they can be prevented. Community mobilisation may be an effective approach. This study aimed to develop an educational package on child injury prevention and assess the feasibility of delivering it with the involvement of women's groups.

Methods An educational package was developed for Female Community Health Volunteers (FCHVs) that included both primary and secondary prevention materials for unintentional child injuries and was adapted from those developed by CIPRB (Bangladesh). Ten women's groups across 9 wards in one village development committee (VDC) area were actively engaged in 6 monthly meetings. The sessions were led by FCHV and lasted for 1-2 hours where rigorous discussions were held using the educational materials provided. Structured checklists were used to obtain feedback and to assess the delivery and reach of the programme.

Results First-aid training, meeting faciliation guide, parents' booklet and educational wall posters were the major outputs of this project. Nine FCHVs received first-aid training and kits. FCHVs convened 10 women's groups to run over 6 months with 25 to 30 mothers attending each session. Each group presented their views on child injury risks and proposed prevention activities at local public meetings. Community members appreciated the project and developed their own interventions to tackle local injury hazards.

Conclusions It is feasible to develop and implement a community mobilisation intervention where women's groups work with FCHVs to prevent injuries in children. The intervention was well received by the women's groups and by community members. 